\title{
Risk Factors of Ectopy of Peripherally Inserted Central Catheter in Obese Patients After Weight Reduction Surgery and Related Nursing Interventions
}

\author{
Li Qiufeng ${ }^{1}$, Huang Guohua ${ }^{1,}$, He Jinai $^{2}$, Liu Yintian ${ }^{3}$ \\ ${ }^{1}$ Department of Intravenous Catheter Nursing Clinic, The First Affiliated Hospital of Jinan University, Guangzhou, China \\ ${ }^{2}$ Department of Nursing, The First Affiliated Hospital of Jinan University, Guangzhou, China \\ ${ }^{3}$ Department of Cardiac Surgery, The First Affiliated Hospital of Jinan University, Guangzhou, China
}

Email address:

jndxhgh@163.com (Huang Guohua)

${ }^{*}$ Corresponding author

\section{To cite this article:}

Li Qiufeng, Huang Guohua, He Jinai, Liu Yintian. Risk Factors of Ectopy of Peripherally Inserted Central Catheter in Obese Patients After Weight Reduction Surgery and Related Nursing Interventions. European Journal of Preventive Medicine. Vol. 8, No. 1, 2020 , pp. 7-11. doi: 10.11648/j.ejpm.20200801.12

Received: February 5, 2020; Accepted: February 17, 2020; Published: February 28, 2020

\begin{abstract}
Objective We analyze risk factors of ectopy of peripherally inserted central catheter tip in obese patients after weight reduction surgery and explore effective nursing interventions. Methods we conducted a retrospective analysis of the general data, PICC data, and position of catheter tip of 190 obese patients who had PICC for weight reduction surgery in our hospital from May 2018 to June 2019. We used monofactor and multi-factor logistic regression analysis to investigate the risk factors of peripherally inserted central catheter tip misplacement in obese patients who underwent weight reduction surgery. Results We found 29 cases $(15.3 \%)$ of ectopy of PICC tip among which 13 cases of misplaced tips were found in internal jugular vein, 5 cases in subclavian vein, 4 cases in brachiocephalic veins, 4 cases in right atrium and 3 cases in axillary vein. The monofactor analysis showed that height, weight, BMI of patients, the vein, arm circumference and experience of operators (how many cases of catheters the operators have inserted) $(\mathrm{P}<0.05)$ were the related risk factors of ectopy of PICC tip in obese patients who underwent weight reduction surgery. The multi-factor logistic regression analysis showed that BMI, arm circumference and experience of operators (how many cases of catheters the operators have inserted) $(\mathrm{P}<0.05)$ were the independent risk factors of ectopy of PICC tip. Conclusions Since BMI, arm circumference and the cases of catheters the operators have inserted are the factors that may cause ectopy of PICC tip, we advise the operators should have good experience of inserting catheters and the cases of catheters they have inserted should be $\geq 100$; Besides, the location of the vein should be determined by ultrasound B before inserting, the length of catheters should be measured by two nurses, and the ectopy of catheter tip can be prevented and corrected by using ultrasonic probe to press the internal jugular vein.
\end{abstract}

Keywords: Obese Patients, Ectopy of Picc, Related Factors, Nursing Interventions

\section{Introduction}

Obesity occurs when adipose tissue is accumulated too much and (or) distributed abnormally in the body and body weight is $20 \%$ higher than standard weight or BMI is over $24 \mathrm{~kg} / \mathrm{m}^{2}[1,2]$.

In recent years, minimally invasive weight reduction surgery is not only effective method for morbid obesity but also develops into a metabolic surgery which becomes a new treatment for refractory obesity combined with diabetes $[3,4]$.
The patients receiving weight reduction surgery are obese and have thick subcutaneous fats. Thus, their blood vessels are less visible and even the large vessels in the upper arm can be seen $2 \sim 3 \mathrm{~cm}$ under the skin under the ultrasound B. Common venipuncture becomes a difficulty for nursing. However, in order for the safety of the patients in operation, construction of venous pathway is required. Because excessive accumulation of subcutaneous fats in the neck and chest causes higher risk of CVC guided by anatomical location, PICC is given prior to weight reduction operation for infusion during or after 
operation.

The ideal location of PICC tip inserted through upper arm is the lower $1 / 3$ of superior vena cava or the joint between superior vena cava and right atrium. When the catheter tip is located outside the superior vena cava, ectopy occurs and the occurrence is $6 \% \sim 10 \%$ [5]. Guidance of ultrasound B can reduce the difficulty in PICC puncture for obese patients, but the weight and body type of them still make the catheterization more difficult, which leads to ectopy of catheter tip. Ectopy of catheter tip induces different complications and thus increases pain and financial burden of patients. Therefore, the current paper analyzes related factors of ectopy of PICC tip in patients undergoing weight reduction surgery and provides relevant nursing strategies. The analysis is reported as follows.

\section{Data and Methods}

\subsection{General Data}

We retrospectively analyzed the data of 190 obese patients (92 males and 98 females aging from 12 to 68, with an average age of $30 \pm 10$ ) who had PICC in weight reduction surgery in our hospital from May 2018 to June 2019. The patients' weight ranges from 67.3 to $232 \mathrm{~kg}$ with an average of $118.1 \pm 30.1 \mathrm{~kg}$, and the BMI from 28.5 to $77.5 \mathrm{~kg} / \mathrm{m} 2$ with an average of $41.63 \pm 8.76 \mathrm{~kg} / \mathrm{m} 2$, arm circumference from 27.5 to $54.5 \mathrm{~cm}$ with an average of $37 \pm 4.8 \mathrm{~cm}$. The 29 patients $(14$ males and 15 females aging from 17 to 57) who had ectopy of catheter tip were assigned to the observation group and the other 161 patients ( 78 males and 84 females aging from 12 to
68) constituted the control group.

\subsection{Methods}

We collected the data including sex, age, height, eight, BMI, the inserted vein of the patients and cases of catheterization of operators. The catheters we used were Bard Access Systems, Per-Q-Cath ${ }^{\mathrm{TM}}$ Pius Catheter.

\subsection{Statistical Methods}

We adopted the statistical software SPSS21.0, using two independent sample $\chi^{2}$ to test enumeration data and two independent sample rank sum test Wilcoxon to analyze ranked data. We used multi-factor logistic regression analysis to deal with the independent risk factors of ectopy of PICC in obese patients who underwent weight reduction surgery. When $\mathrm{P}<0.05$, there was statistical significance $(\alpha=0.05)$.

\section{Results}

\subsection{Location Through Chest X-ray for PICC in 190 Obese Patients Receiving Weight Reduction Surgery}

There were $29(15.3 \%)$ cases of ectopy of catheter tip among which 13 cases of misplaced tips were found in internal jugular vein, 5 cases in subclavian vein, 4 cases in brachiocephalic veins, 4 cases in right atrium and 3 cases in axillary vein. The data are shown in the Table 1.

Table 1. Location of Catheter Tip after PICC in Obese Patients Having Received Weight Reduction Surgery n (\%).

\begin{tabular}{lll}
\hline Location of catheter tip & Observation group n=29 & Control group n=161 \\
\hline Ectopy of catheter & & \\
internal jugular vein & $13(44.83)$ & $0(0.00)$ \\
subclavian vein & $5(17.24)$ & $0(0.00)$ \\
brachiocephalic vein & $4(13.79)$ & $0(0.00)$ \\
right atrium & $4(13.79)$ & $0(0.00)$ \\
axillary vein & $3(10.34)$ & $0(0.00)$ \\
Right position of catheter & $0(0.00)$ & $161(100)$ \\
Lower $1 / 3$ of superior vena cava (T5-T7) & & \\
\hline
\end{tabular}

\subsection{Variable Assignment Table of Regression Analysis}

We used monofactor analysis and multi-factor logistic regression analysis to analyze factors associated with ectopy of catheter tip. The variable assignment of regression analysis is shown in the following Table 2.

Table 2. Variable Assignment Table of Regression Analysis.

\begin{tabular}{ll}
\hline Variables & Assigned value \\
\hline Sex & Female $\rightarrow 0$, Male $\rightarrow 1$ \\
Age & $\leq 60 \rightarrow 0,>60 \rightarrow 1$ \\
Height & $\leq 170 \mathrm{~cm} \rightarrow 0,>170 \mathrm{~cm} \rightarrow 1$ \\
Weight & $\leq 100 \mathrm{~kg} \rightarrow 0,>100 \mathrm{~kg} \rightarrow 1$ \\
BMI & $28 \leq \mathrm{BMI}<30 \rightarrow 0,30 \leq \mathrm{BMI}<40 \rightarrow 1$, BMI $\geq 40 \rightarrow 2$ \\
Arm circumference & $\leq 30 \mathrm{~cm} \rightarrow 0,>30 \mathrm{~cm} \rightarrow 1$ \\
Inserted vein & Left basilic vein $\rightarrow 0$, Right basilic vein $\rightarrow 1$, left brachial vein $\rightarrow 2$, Left median cubital vein $\rightarrow 3$ \\
Cases of inserting catheters & $\leq 100$ cases $\rightarrow 0, \geq 100$ cases $\rightarrow 1$ \\
\hline
\end{tabular}

\subsection{Monofactor Analysis of Ectopy of PICC in Obese Patients Having Received Weight Reduction Surgery}

The monofactor analysis found that the weight, BMI, arm circumference of the patients, inserted vein and cases of inserting 
catheters were the related factors of ectopy of PICC in obese patients having received weight reduction surgery $(\mathrm{P}<0.05)$ as shown in the Table 3.

Table 3. Monofactor Analysis of Related Factors of Ectopy of PICC in Obese Patients Having Received Weight Reduction Surgery.

\begin{tabular}{|c|c|c|c|c|}
\hline Variables & Observation group $(n=29)$ & Control group $(n=161)$ & Statistics & $\mathbf{P}$ \\
\hline Sex & & & $0.000^{\#}$ & 0.986 \\
\hline Male & $14(48.28)$ & $83(51.85)$ & & \\
\hline Female & $15(51.72)$ & $83(51.85)$ & & \\
\hline Age & & & $3.812^{\#}$ & 0.051 \\
\hline$\leq 60$ & $27(93.10)$ & $157(97.52)$ & & \\
\hline$>60$ & $2(6.90)$ & $4(2.48)$ & & \\
\hline Height & & & $1.399^{\#}$ & 0.237 \\
\hline$>170 \mathrm{~cm}$ & $8(27.59)$ & $63(39.13)$ & & \\
\hline Weight & & & $6.911^{\#}$ & 0.009 \\
\hline$\leq 100 \mathrm{~kg}$ & $10(34.48)$ & $63(39.13)$ & & \\
\hline$>100 \mathrm{~kg}$ & $19(65.52)$ & $98(60.87)$ & & \\
\hline BMI & & & $-3.332^{\#}$ & 0.001 \\
\hline I in obesity $28-30$ & $1(3.45)$ & $6(3.73)$ & & \\
\hline II in obesity $30-40$ & $5(17.24)$ & $80(49.69)$ & & \\
\hline III in obesity $\geq 40$ & $23(79.31)$ & $75(46.58)$ & & \\
\hline$\leq 30 \mathrm{~cm}$ & 0 & $13(8.07)$ & & \\
\hline$>30 \mathrm{~cm}$ & $29(100)$ & $148(91.93)$ & & \\
\hline Inserted vein & & & $-2.745^{*}$ & 0.006 \\
\hline Left basilic vein & $22(75.86)$ & $149(92.55)$ & & \\
\hline Right basilic vein & $3(10.34)$ & $6(3.73)$ & & \\
\hline left brachial vein & $2(6.90)$ & $3(1.86)$ & & \\
\hline Left median cubital vein & $2(6.90)$ & $3(1.86)$ & & \\
\hline Cases of inserting catheters & & & $23.448 \#$ & 0.002 \\
\hline$\leq 100$ cases & $18(62.07)$ & $51(31.68)$ & & \\
\hline$>100$ cases & $11(37.93)$ & $110(68.32)$ & & \\
\hline
\end{tabular}

Note: \# means $\chi^{2}$ value, * means u value.

\subsection{Multi-factor Logistic Regression Analysis of Independent Risk Factors of Ectopy of PICC in Obese Patients Having Received Weight Reduction Surgery}

The multi-factor logistic regression analysis found that
BMI, arm circumference of the patients and cases of inserting catheters of the operators were the independent risk factors of ectopy of PICC in obese patients having received weight reduction surgery as shown in the following Table 4.

Table 4. Multi-factor Logistic Regression Analysis of Independent Risk Factors of Ectopy of PICC in Obese Patients Having Received Weight Reduction Surgery.

\begin{tabular}{llllll}
\hline Variables & $\boldsymbol{\beta}$ & S. E & P & OR & 95\%CI \\
\hline BMI & 1.878 & 0.549 & 0.006 & 6.539 & $(2.229,19.188)$ \\
Arm circumference & -0.954 & 0.448 & 0.034 & 0.385 & $(0.160,0.928)$ \\
Cases of inserting catheters & -2.161 & 0.523 & 0.000 & 0.115 & $(0.041,0.321)$ \\
\hline
\end{tabular}

\section{Discussion}

Obesity is associated with diabetes, high pressure, coronary heart disease, sleep apnea and many other health, social and psychological problems which threaten human health [6]. Weight reduction surgery is the longest effective treatment for obesity and its complications so far [7-9]. PICC is an important pathway for the protection of blood vessels, intraoperative treatment and postoperative infusion in weight reduction surgery for obese patients. However, PICC-related complications such as ectopy of catheter tip, mechanical phlebitis and venous thrombosis are severe problems. Among them, the ectopy of catheter tip is the most common and affects the effect of PICC. The current analysis finds 29 cases of ectopy of PICC catheter tip in 190 obese patients, accounting for $15.26 \%$. Monofactor analysis shows that weight, BMI, arm circumference of the patients, inserted vein and cases of catheters the operators have inserted are the related risk factors of ectopy of PICC catheter tip in obese patients who have received weight reduction surgery. The multi-factor logistic regression analysis shows that MBI, arm circumference of the patients and cases of catheters the operators have inserted are the independent risk factors of ectopy of PICC catheter tip.

\subsection{Analysis of Association Between Independent Risk Factors and Ectopy of PICC Catheter Tip}

\subsubsection{BMI}

Among the 29 cases of ectopy of PICC tip, the cases of BMI over $30 \mathrm{~kg} / \mathrm{m}^{2}$ account for $96.55 \%$. In the neck, chest and armpit of 21 patients, the subcutaneous fats are abnormally 
accumulated which presses the local blood vessels, leading to deformation and narrowing of blood vessels. As a result, it is difficult to insert catheters to the vessels resulting 5 cases of catheter misplaced in subclavian vein, 3 cases in axillary vein and 13 cases in internal jugular vein. Because it is hard to turn the head towards the puncture side and touch the shoulder, internal jugular vein cannot be compressed causing the ectopy of catheter tip.

\subsubsection{Arm Circumference}

The arm circumference of 29 patients in the observation group are over $30 \mathrm{~cm}$ and their subcutaneous fats thicken. Only the large vessels $2-3 \mathrm{~cm}$ under the skin can be seen under the guidance of the ultrasound. The thickness of subcutaneous fats is hardly considered when predicting the length of catheter prior to catheterization. As a result, the catheter cannot be sent to its right position. In the observation group, 4 cases of catheter tips only reach brachiocephalic vein.

\subsubsection{Cases of Catheters Operators Have Inserted}

Research [10] shows that the operator inserting the catheters should have inserted at least 10 cases of catheters independently and no catheterization-related adverse events have occurred. The success rate of PICC catheterization depends on the operators' skill and experience [11]. As we all know, PICC catheterization is an invasive operation and has unpredictable risks [12, 13]. Hence, identification and measures of the risks are positively correlated with the cases of catheters the operators have inserted. Among the 29 cases of ectopy of catheters in the observation group, 18 cases $(62.07 \%)$ of catheters are inserted by operators who have inserted catheters less than 100 cases before.

\subsection{Nursing Strategies}

\subsubsection{A New Method to Determine the Length of Catheters}

In this study, logistic regression analysis shows that arm circumference is an independent risk factor of ectopy of the PICC catheter tip. The length of the inserted catheter needs to be measured before PICC catheterization. The method is to measure the distance from the puncture point to the right sternoclavicular joint and then back to the third intercostal space at the right edge of the sternum. Because of the thick subcutaneous fat in obese patients, there are two difficulties in determining the length of the catheter. One is the inaccurate anatomical positioning: the third intercostal space is not obvious in obese patients, so it is difficult to determine its location. Estimated location results in a much longer catheter. In the observation group, 4 cases of catheter tips are inserted into the right atrium. The other difficulty is that the third intercostal space can be detected but the length of the catheter determined by the location of the third intercostal space is shorter than the actually inserted length [14]. In summary, when determining the length of catheter for obese patients, the thickness of the subcutaneous fat between the puncture point and the puncture vessels plus $5 \mathrm{~cm}$ is equivalent to the length from the sternoclavicular joint to the third intercostal space at the right edge of the sternum. Among the 148 patients in the control group whose arm circumference are more than $30 \mathrm{~cm}$, the length of the inserted catheter is determined in this way, and the catheter tip can reach the right position.

\subsubsection{Reducing Resistance to Catheter Insertion Via Change of Upper Limb Position}

The patients with BMI over $30 \mathrm{~kg} / \mathrm{m}^{2}$ in the observation group account for more than $96.55 \%$. Excessive accumulation of subcutaneous fats in the neck, chest, axilla, etc., compress local blood vessels, causing deformation and narrowing of the patient's blood vessels. Anatomically, changes in the position of the shoulder affect the condition of the subclavian vein. The patients need to change the position of their upper limbs during different stages of insertion so that the catheters can be successfully inserted into the right location. In the control group, when the catheter tip is at the head of the subclavian vein under the ultrasound $B$ scan, the patients need to move their limbs $5 \mathrm{~cm}$ up towards head from abduction at 90 degrees and the catheter is inserted $3 \mathrm{~cm}$ deeper, and then the patients should be assisted to adduct their upper limbs $10 \mathrm{~cm}$ closer towards their legs in order to reduce the pressure of the subcutaneous fats in the chest on blood vessels so that the catheter can pass through the subclavian vein. In the control group, among patients with a BMI $\geq 40 \mathrm{~kg} / \mathrm{m}^{2}$ there are less ectopic catheter tips than those in the observation group.

\subsubsection{Cases of Catheters Operators Have Inserted}

The success rate of PICC puncture depends on the operator's skills and proficiency. The cases of catheters an operator has inserted and his/her skill are proportional to the occurrence of risks during the catheterization [12, 15-17]. In this study, operators who have inserted more than 100 cases of catheters have less ectopy of catheter tip in their catheterization for obese patients. In the observation group, $62.07 \%$ of ectopic catheter tips are done by operators who have inserted catheters less than 100 cases and the other $37.93 \%$ of the ectopic catheters are performed by operators who have inserted more than 100 cases of catheters. Therefore, we suggest that the skill and experience of operators should be taken into consideration (More than 100 cases are appropriate) when performing PICC catheterization in obese patients.

\section{Conclusions}

In summary, when operators have the experience of inserting over 100 cases of catheters and can apply their puncture skill and techniques to overcome difficulties and eliminate risks during the catheterization for obese patient, the length of the catheter is determined by two people via the above new method, and the patients have received instructions on changes of limb position during catheterization to reduce the difficulty of inserting the catheter due to the compression of the blood vessels by accumulation of subcutaneous fats, the occurrence of ectopy of PICC catheter tip can be reduced. Accordingly, intraoperative and postoperative infusion of obese patients can be ensured. Therefore, these nursing strategies are worthy of clinical application. 


\section{References}

[1] Eun Young Lee. (2018). Epidemic obesity in children and adolescents: risk factors and prevention. Frontiers of Medicine, $12(6), 658-666$.

[2] Ahuja, N. K, \& Nimgaonkar, A. (2016). Precision bariatrics: toward a new paradigm of personalized devices in obesity therapeutics. Obesity Surgery, 26 (7), 1642-1645.

[3] Dillon, C, Peddle, J. Twells, L., Lester, K., \& Gregory, D. (2015). Rapid reduction in use of antidiabetic medication after laparoscopic sleeve gastrectomy: the new found land and labrador bariatric surgery cohort (basco) study. The Canadian journal of hospital pharmacy, 68 (2), 113-120.

[4] Sheu, E. G., Channick, R., \& Gee, D. W. (2016). Improvement in severe pulmonary hypertension in obese patients after laparoscopic gastric bypass or sleeve gastrectomy. Surgical Endoscopy, 30 (2), 633-637.

[5] J. J. Menéndez, C. Verdú, B. Calderón, A. Gómez-Zamora, C. Schüffelmann, \& Cruz, J. J., et al. (2016). Incidence and risk factors of superficial and deep vein thrombosis associated with peripherally inserted central catheters in children. Journal of Thrombosis and Haemostasis, 14 (11), 2158-2168.

[6] Patel, S. R., Malhotra, A., White, D. P., Gottlieb, D. J., \& Hu, F. B. (2006). Association between reduced sleep and weight gain in women. American Journal of Epidemiology, 164 (10), 947-954.

[7] Listed, N. A. (2008). Surgery for obesity in adults. Drug \& Therapeutics Bulletin, 46 (6), 41-5.

[8] Zitsman, J. L., Digiorgi, M. F., Marr, J. R., Witt, M. A., \& Bessler, M. (2011). Comparative outcomes of laparoscopic adjustable gastric banding in adolescents and adults. Surgery for Obesity and Related Diseases, 7 (6), 720-726.

[9] Colquitt, J. L., Pickett, K., Loveman, E., \& Frampton, G. K. (2014). Surgery for weight loss in adults. Cohrane Database of
Systematic Reviews, 8 (Issue 8 Art. No.: CD003641), CD003641.

[10] Kang, J. R., Long, L. H., Yan, S. W., Wei, W. W., Jun, H. Z., \& Chen, W. (2017). Peripherally inserted central catheter-related vein thrombosis in patients with lung cancer. Clin Appl Thromb Hemost, 23 (2), 181-186.

[11] Safdar, N., \& Maki, D. G. (2005). Risk of catheter-related bloodstream infection with peripherally inserted central venous catheters used in hospitalized patients. Chest, 128 (2), 489-495.

[12] Cotogni, P., Barbero, C., Garrino, C., Degiorgis, C., Mussa, B., $\&$ De Francesco, A., et al. (2015). Peripherally inserted central catheters in non-hospitalized cancer patients: 5-year results of a prospective study. Supportive Care in Cancer, 23 (2), 403-409.

[13] Cotogni, P., Pittiruti, M., Barbero, C., Monge, T., Palmo, A., \& Boggio Bertinet, D. (2013). Catheter-related complications in cancer patients on home parenteral nutrition: a prospective study of over 51,000 catheter days. Journal of Parenteral and Enteral Nutrition, 37 (3), 375-383.

[14] Tracey L. Abitz. (2016). Cultural congruence and infusion nursing practice. Journal of Infusion Nursing the Official Publication of the Infusion Nurses Society, 39 (2), 75-79.

[15] Botella-Carretero, J. I., Carrero, C., Guerra, E., Valbuena, B., Arrieta, F., \& Calanas, A., et al. (2013). Role of peripherally inserted central catheters in home parenteral nutrition: a 5 -year prospective study. Journal of Parenteral and Enteral Nutrition, 37 (4), 544-549.

[16] Opilla, \& Marianne. (2017). Peripherally inserted central catheter experience in long-term home parenteral nutrition patients. Journal of the Association for Vascular Access, 22 (1), $42-45$

[17] Line Dahlstrøm Christensen, Henrik Højgaard Rasmussen, \& Vinter-Jensen, L. (2013). Peripherally inserted central catheter for use in home parenteral nutrition: a 4-year follow-up study. Journal of Parenteral and Enteral Nutrition, 38 (8), 1003-6. 